\title{
AN EFFICIENT PARAMETERIZATION FOR PARETO-OPTIMAL BEAMFORMERS FOR $K$-USER MIMO INTERFERENCE CHANNELS
}

\author{
Juho Park and Youngchul Sung \\ Dept. of Electrical Engineering, KAIST, Daejeon, 305-701, South Korea. \\ Email:\{jhp@, ysung@ee.\}kaist.ac.kr
}

\begin{abstract}
In this paper, Pareto-optimal beamforming in the $K$-pair Gaussian multiple-input multiple-output (MIMO) interference channel is considered. Under the assumption of Gaussian signaling at transmitters and single-user decoding at receivers, a necessary condition for any transmit signal covariance matrix to achieve a Pareto boundary point of the achievable rate region is derived. Based on the necessary condition for Pareto-optimality, an efficient parameterization for Pareto-optimal transmit signal covariance matrices is obtained. The obtained parameter space is given by the product manifold of a Stiefel manifold and a subset of a hyperplane, which is a low dimensional embedded submanifold of the original high dimensional beam search space. The new parameterization enables us to devise very efficient beam design algorithms for the $K$-pair MIMO interference channel.
\end{abstract}

Index Terms- Interference Channels, Multiple-Input MultipleOutput, Pareto-optimality, Transmit Signal Covariance Matrices, Stiefel Manifolds

\section{INTRODUCTION}

Due to the importance of proper interference control in current and future wireless systems, multi-user MIMO interference channels have gained much interest from the research community recently. One of the meaningful approaches to the interference channel problem is the game-theoretical approach which investigates Paretooptimality of coordinated beamforming in multi-user MIMO interference channels. Many results are now available on efficient parameterization and beam design for Pareto-optimal beamforming in multiple-input single-output (MISO) interference channels [2-8]. It is known that for MISO interference channels, any Pareto-optimal transmit beam vector at a transmitter is a normalized convex combination of the zero-forcing beam and the matched-filtering beam in the case of two users, and a linear combination of the channel vectors from the transmitter to all receivers in the case of a general number of users [2]. However, not many results are available on the Pareto-optimal beam structure and design for MIMO interference channels yet, although some results are available for limited circumstances [9-11].

In this paper, we investigate the Pareto-optimal beamforming problem in MIMO interference channels and provide a necessary condition for Pareto-optimal beamformers for general MIMO interference channels. It is shown that any Pareto-optimal transmit signal

This research was supported by Basic Science Research Program through the National Research Foundation of Korea (NRF) funded by the Ministry of Education, Science and Technology (2010-0021269). The journal version of this paper was submitted and is available at http://arxiv.org/abs/1211.4213 [1]. covariance matrix at a transmitter should have its column space contained in the union of the eigen-spaces of the channel matrices from the transmitter to all receivers. Based on the obtained necessary condition, an efficient parameterization for the beam search space not losing Pareto-optimality is derived. The derived beam search space or parameter space is given by a low dimensional submanifold embedded in the original high dimensional beam search space and described by the product manifold of a Stiefel manifold and $a$ subset of a hyperplane. Furthermore, the dimension of the proposed beam search space is independent of the number of transmit antennas when the number of transmit antennas is large and thus the proposed parameterization is very useful for coordinated beamforming in upcoming wireless networks adopting massive MIMO technologies.

\subsection{Notation}

We will make use of standard notational conventions. Vectors and matrices are written in boldface with matrices in capitals. All vectors are column vectors. For a matrix $\mathbf{A}, \mathbf{A}^{H},\|\mathbf{A}\|, \operatorname{tr}(\mathbf{A})$, and $|\mathbf{A}|$ indicate the Hermitian transpose, 2-norm, trace, and determinant of $\mathbf{A}$, respectively. $\mathcal{C}(\mathbf{A})$ denotes the column space of $\mathbf{A}$ and $\mathcal{C}^{\perp}(\mathbf{A})$ denotes the orthogonal complement of $\mathcal{C}(\mathbf{A})$. For matrices $\mathbf{A}$ and $\mathbf{B}$, $\mathbf{A} \succcurlyeq \mathbf{B}$ means that $\mathbf{A}-\mathbf{B}$ is positive semi-definite. $\mathbb{S}_{+}^{n \times n}$ denotes the set of positive semi-defnite matrices of size $n \times n$. $\mathbf{I}_{n}$ stands for the identity matrix of size $n$ (the subscript is omitted when unnecessary). $\mathrm{x} \sim \mathcal{C} \mathcal{N}(\boldsymbol{\mu}, \boldsymbol{\Sigma})$ means that $\mathrm{x}$ is circularly-symmetric complex Gaussian-distributed with mean vector $\boldsymbol{\mu}$ and covariance matrix $\boldsymbol{\Sigma}$.

\section{SYSTEM MODEL}

In this paper, we consider the $K$-pair Gaussian MIMO interference channel where every transmitter is assumed to have $N$ transmit antennas and receiver $i$ is assumed to have $M_{i}(\geq 1)$ receive antennas. We will call the considered MIMO interference channel the $K$-pair Gaussian $\left(N, M_{1}, \cdots, M_{K}\right)$ MIMO interference channel. The received signal at receiver $i$ is given by

$$
y_{i}=\mathbf{H}_{i i} \mathbf{s}_{i}+\sum_{j=1, j \neq i}^{K} \mathbf{H}_{i j} \mathbf{s}_{j}+\mathbf{n}_{i},
$$

where $\mathbf{H}_{i j}$ denotes the $M_{i} \times N$ channel matrix from transmitter $j$ to receiver $i$; $\mathbf{s}_{j}$ is the $N \times 1$ transmit signal vector generated from a Gaussian codebook with distribution $\mathcal{C N}\left(0, \mathbf{Q}_{j}\right)$; and $\mathbf{n}_{i}$ is the additive noise vector from $\mathcal{C N}(0, \mathbf{I})$. The transmit signal covariance matrix $\mathbf{Q}_{i}:=\mathbb{E}\left\{\mathbf{s}_{i} \mathbf{s}_{i}^{H}\right\}, i=1, \cdots, K$, is selected from the feasible set

$\mathcal{Q}_{i}:=\left\{\mathbf{Q} \in \mathbb{S}_{+}^{N \times N}: \operatorname{tr}(\mathbf{Q}) \leq P_{i}, 1 \leq \operatorname{rank}(\mathbf{Q}) \leq \min \left\{M_{i}, N\right\}\right\}$. 
The rank constraint in (2) guarantees that the number of data streams is between one and the maximum, $\min \left\{M_{i}, N\right\}$.

For a given channel realization with single-user decoding receivers, i.e., with interference treated as noise, the achievable rate region is defined as the union of rate tuples that can be achieved by all possible combinations of transmit signal covariance matrices:

$$
\begin{aligned}
& \mathcal{R}:=\bigcup_{\left\{\mathbf{Q}_{i}: \mathbf{Q}_{i} \in \mathcal{Q}_{i}, 1 \leq i \leq K\right\}}\left(R_{1}\left(\mathbf{Q}_{1}, \cdots, \mathbf{Q}_{K}\right), \cdots, R_{K}\left(\mathbf{Q}_{1}, \cdots, \mathbf{Q}_{K}\right)\right) \\
&
\end{aligned}
$$

where

$$
\begin{aligned}
& R_{i}\left(\mathbf{Q}_{1}, \cdots, \mathbf{Q}_{K}\right) \\
& \quad=\log _{2}\left|\mathbf{I}+\left(\mathbf{I}+\sum_{j \neq i} \mathbf{H}_{i j} \mathbf{Q}_{j} \mathbf{H}_{i j}^{H}\right)^{-1} \mathbf{H}_{i i} \mathbf{Q}_{i} \mathbf{H}_{i i}^{H}\right|
\end{aligned}
$$

for $i=1, \cdots, K$. The outer boundary of the achievable rate region $\mathcal{R}$ is called the Pareto boundary of $\mathcal{R}$. The Pareto boundary consists of rate-tuples for which the rate of one user cannot be increased without decreasing the rate of at least one other user.

\section{A NECESSARY CONDITION FOR PARETO-OPTIMALITY}

In this section, we present a necessary condition for Pareto-optimal transmit covariance matrices for the $K$-pair Gaussian $\left(N, M_{1}, \cdots\right.$, $\left.M_{K}\right)$ MIMO interference channel with single-user decoding receivers, which is given in the following theorem.

Theorem 1 For the $K$-pair Gaussian $\left(N, M_{1}, \cdots, M_{K}\right)$ MIMO interference channel in which the channel matrices $\left\{\mathbf{H}_{i j}\right\}$ are randomly realized and interference is treated as noise at each receiver, any Pareto-optimal transmit signal covariance matrix $\mathbf{Q}_{i}^{\star}$ at transmitter $i$ should satisfy

$$
\mathcal{C}\left(\mathbf{Q}_{i}^{\star}\right) \subseteq \mathcal{C}\left(\left[\mathbf{H}_{1 i}^{H}, \cdots, \mathbf{H}_{K i}^{H}\right]\right) \quad \text { in all cases }
$$

and

$$
\operatorname{tr}\left(\mathbf{Q}_{i}^{\star}\right)=P_{i} \text { in the case that } N \geq \sum_{i=1}^{K} M_{i}
$$

Proof: We first consider the case of $N \geq \sum_{i=1}^{K} M_{i}$. Suppose that $\left[\mathbf{H}_{1 i}^{H}, \cdots, \mathbf{H}_{K i}^{H}\right] \in \mathbb{C}^{N \times \sum_{i} M_{i}}$ is of rank $m(<N)$. (When $m=N,(5)$ holds trivially since $\mathcal{C}\left(\left[\mathbf{H}_{1 i}^{H}, \cdots, \mathbf{H}_{K i}^{H}\right]\right)=$ $\mathbb{C}^{N}$.) Then, there exists an orthonormal basis $\left\{\mathbf{u}_{l}\right\}_{l=1}^{N-m}$ that spans $\mathcal{C}^{\perp}\left(\left[\mathbf{H}_{1 i}^{H}, \cdots, \mathbf{H}_{K i}^{H}\right]\right)$ :

$$
\mathcal{C}\left(\left\{\mathbf{u}_{l}\right\}_{l=1}^{N-m}\right)=\mathcal{C}^{\perp}\left(\left[\mathbf{H}_{1 i}^{H}, \cdots, \mathbf{H}_{K i}^{H}\right]\right) .
$$

Suppose that a set $\left\{\mathbf{Q}_{i}: i=1, \cdots, K\right\}$ of transmit covariance matrices achieves a Pareto-boundary point of $\mathcal{R}$ and $\mathcal{C}\left(\mathbf{Q}_{i}\right) \nsubseteq$ $\mathcal{C}\left(\left[\mathbf{H}_{1 i}^{H}, \cdots, \mathbf{H}_{K i}^{H}\right]\right)$ at transmitter $i$. Then, $\mathbf{Q}_{i}$ is expressed as

$$
\mathbf{Q}_{i}=\left[\mathbf{H}_{1 i}^{H}, \cdots, \mathbf{H}_{K i}^{H}\right] \mathbf{X}_{i}\left[\mathbf{H}_{1 i}^{H}, \cdots, \mathbf{H}_{K i}^{H}\right]^{H}+\sum_{l=1}^{N-m} \alpha_{l}^{2} \mathbf{u}_{l} \mathbf{u}_{l}^{H},
$$

where $\mathbf{X}_{i} \succcurlyeq \mathbf{0}, \operatorname{tr}\left(\mathbf{Q}_{i}\right) \leq P_{i}$, and $\alpha_{l}^{2}=\mathbf{u}_{l}^{H} \mathbf{Q}_{i} \mathbf{u}_{l} . \mathcal{C}\left(\mathbf{Q}_{i}\right) \nsubseteq$ $\mathcal{C}\left(\left[\mathbf{H}_{1 i}^{H}, \cdots, \mathbf{H}_{K i}^{H}\right]\right)$ implies $\alpha_{l}^{2} \neq 0$ for some $l$. Let $\hat{i}$ be such an index and construct a transmit signal covariance matrix as

$$
\mathbf{Q}_{i}^{\prime}=\mathbf{Q}_{i}-\alpha_{\hat{i}}^{2} \mathbf{u}_{i} \mathbf{u}_{\hat{i}}^{H} .
$$

Then, $\operatorname{tr}\left(\mathbf{Q}_{i}^{\prime}\right)=\operatorname{tr}\left(\mathbf{Q}_{i}\right)-\alpha_{\hat{i}}^{2}<\operatorname{tr}\left(\mathbf{Q}_{i}\right) \leq P_{i}$ and $\mathbf{Q}_{i}^{\prime} \succcurlyeq \mathbf{0} .{ }^{1}$ Hence, $\mathbf{Q}_{i}^{\prime}$ is a valid transmit signal covariance matrix. Now, we consider the rate-tuple achieved by $\left\{\mathbf{Q}_{1}, \cdots, \mathbf{Q}_{i}^{\prime}, \cdots, \mathbf{Q}_{K}\right\}$. For this, we denote the interference covariance matrix at receiver $i$ by

$$
\boldsymbol{\Phi}_{i}:=\mathbf{I}+\sum_{k \neq i} \mathbf{H}_{i k} \mathbf{Q}_{k} \mathbf{H}_{i k}^{H} .
$$

Then, the rate at user $i$ is given by

$$
\begin{aligned}
& R_{i}\left(\mathbf{Q}_{1}, \cdots, \mathbf{Q}_{i}^{\prime}, \cdots, \mathbf{Q}_{K}\right) \\
& =\log _{2}\left|\mathbf{I}+\boldsymbol{\Phi}_{i}^{-1} \mathbf{H}_{i i} \mathbf{Q}_{i}^{\prime} \mathbf{H}_{i i}^{H}\right| \\
& =\log _{2}\left|\mathbf{I}+\boldsymbol{\Phi}_{i}^{-1} \mathbf{H}_{i i}\left(\mathbf{Q}_{i}-\alpha_{\hat{i}}^{2} \mathbf{u}_{i} \mathbf{u}_{i}^{H}\right) \mathbf{H}_{i i}^{H}\right| \\
& \stackrel{(a)}{=} \log _{2}\left|\mathbf{I}+\boldsymbol{\Phi}_{i}^{-1} \mathbf{H}_{i i} \mathbf{Q}_{i} \mathbf{H}_{i i}^{H}\right| \\
& =R_{i}\left(\mathbf{Q}_{1}, \cdots, \mathbf{Q}_{i}, \cdots, \mathbf{Q}_{K}\right),
\end{aligned}
$$

where $(a)$ holds since $\mathbf{u}_{\hat{i}} \in \mathcal{C}^{\perp}\left(\left[\mathbf{H}_{1 i}^{H}, \cdots, \mathbf{H}_{K i}^{H}\right]\right)$ and hence $\mathbf{H}_{i i} \mathbf{u}_{\hat{i}}=0$. Similarly, the rate of user $j(\neq i)$ is given by

$$
\begin{aligned}
& R_{j}\left(\mathbf{Q}_{1}, \cdots, \mathbf{Q}_{i}^{\prime}, \cdots, \mathbf{Q}_{K}\right) \\
& =\log _{2}\left|\mathbf{I}+\left(\mathbf{I}+\sum_{k \neq j, k \neq i} \mathbf{H}_{j k} \mathbf{Q}_{k} \mathbf{H}_{j k}^{H}+\mathbf{H}_{j i} \mathbf{Q}_{i}^{\prime} \mathbf{H}_{j i}^{H}\right)^{-1} \mathbf{H}_{j j} \mathbf{Q}_{j} \mathbf{H}_{j j}^{H}\right| \\
& =\log _{2}\left|\mathbf{I}+\left(\boldsymbol{\Phi}_{j}-\alpha_{\hat{i}}^{2} \mathbf{H}_{j i} \mathbf{u}_{i} \mathbf{u}_{i}^{H} \mathbf{H}_{j i}^{H}\right)^{-1} \mathbf{H}_{j j} \mathbf{Q}_{j} \mathbf{H}_{j j}^{H}\right| \\
& \stackrel{(b)}{=} \log _{2}\left|\mathbf{I}+\mathbf{\Phi}_{j}^{-1} \mathbf{H}_{j j} \mathbf{Q}_{j} \mathbf{H}_{j j}^{H}\right| \\
& =R_{j}\left(\mathbf{Q}_{1}, \cdots, \mathbf{Q}_{i}, \cdots, \mathbf{Q}_{K}\right),
\end{aligned}
$$

where $(b)$ holds since $\mathbf{u}_{\hat{i}} \in \mathcal{C}^{\perp}\left(\left[\mathbf{H}_{1 i}^{H}, \cdots, \mathbf{H}_{K i}^{H}\right]\right)$. Thus, the ratetuple is unchanged with $\left\{\mathbf{Q}_{1}, \cdots, \mathbf{Q}_{i}^{\prime}, \cdots, \mathbf{Q}_{K}\right\}$.

Now, we construct another transmit covariance matrix $\mathbf{Q}_{i}^{\prime \prime}$ as

$$
\mathbf{Q}_{i}^{\prime \prime}=\mathbf{Q}_{i}^{\prime}+\delta \mathbf{v} \mathbf{v}^{H}
$$

where $\mathbf{v}$ satisfies $\mathbf{H}_{i i} \mathbf{v} \neq \mathbf{0}$ while $\mathbf{H}_{j i} \mathbf{v}=\mathbf{0}$ for all $j \neq i$, i.e., $\mathbf{v} \in$ $\mathcal{C}\left(\mathbf{H}_{i i}^{H}\right) \cap\left(\bigcup_{j \neq i} \mathcal{C}\left(\mathbf{H}_{j i}^{H}\right)\right)^{\perp}$. Such $\mathbf{v}$ almost surely exists since the event $\mathcal{C}\left(\mathbf{H}_{i i}^{H}\right) \subseteq \bigcup_{j \neq i} \mathcal{C}\left(\mathbf{H}_{j i}^{H}\right)$ has measure zero for randomly realized channel matrices. (Note that the dimension of $\bigcup_{j \neq i} \mathcal{C}\left(\mathbf{H}_{j i}^{H}\right)$ is at most $\sum_{j \neq i} M_{j}$ which is strictly less than $N$ by the assumption $\sum_{i} M_{i} \leq$ $N$.) Here, $\delta>0$ is chosen to be $\delta \leq \frac{1}{\operatorname{tr}\left(\mathbf{v v}^{H}\right)}\left(P_{i}-\operatorname{tr}\left(\mathbf{Q}_{i}^{\prime}\right)\right)$ so that

$$
\operatorname{tr}\left(\mathbf{Q}_{i}^{\prime \prime}\right)=\operatorname{tr}\left(\mathbf{Q}_{i}^{\prime}+\delta \mathbf{v} \mathbf{v}^{H}\right) \leq \operatorname{tr}\left(\mathbf{Q}_{i}^{\prime}\right)+\left(P_{i}-\operatorname{tr}\left(\mathbf{Q}_{i}^{\prime}\right)\right)=P_{i}
$$

Denote the interference covariance matrix at receiver $j$ with the set $\left\{\mathbf{Q}_{1}, \cdots, \mathbf{Q}_{i}^{\prime}, \cdots, \mathbf{Q}_{K}\right\}$ of transmit covariance matrices by

$$
\Psi_{j}=\mathbf{I}+\sum_{k \neq j, k \neq i} \mathbf{H}_{j k} \mathbf{Q}_{k} \mathbf{H}_{j k}^{H}+\mathbf{H}_{j i} \mathbf{Q}_{i}^{\prime} \mathbf{H}_{j i}^{H} .
$$

${ }^{1} \mathbf{Q}_{i}^{\prime} \succcurlyeq 0$ can be shown by a similar argument to that in [12]. First, by the definition of $\alpha_{\hat{i}}^{2}$ and the fact $\left\|\mathbf{u}_{l}\right\|=1, \mathbf{u}_{\hat{i}}^{H} \mathbf{Q}_{i}^{\prime} \mathbf{u}_{\hat{i}}=\mathbf{u}_{\hat{i}}^{H}\left(\mathbf{Q}_{i}-\right.$ $\left.\alpha_{\hat{i}}^{2} \mathbf{u}_{\hat{i}} \mathbf{u}_{\hat{i}}^{H}\right) \mathbf{u}_{\hat{i}}=\mathbf{u}_{\hat{i}}^{H} \mathbf{Q}_{i} \mathbf{u}_{\hat{i}}-\alpha_{\hat{i}}^{2}\left\|\mathbf{u}_{\hat{i}}\right\|^{2}=0$. For any vector $\mathbf{w}$ such that $\mathbf{w}^{H} \mathbf{u}_{\hat{i}}=0$, we have $\mathbf{w}^{H} \mathbf{Q}_{i}^{\prime} \mathbf{w}=\mathbf{w}^{H}\left(\mathbf{Q}_{i}-\alpha_{\hat{i}}^{2} \mathbf{u}_{\hat{i}} \mathbf{u}_{\hat{i}}^{H}\right) \mathbf{w}=\mathbf{w}^{H} \mathbf{Q}_{i} \mathbf{w} \geq$ 0 since $\mathbf{Q}_{i}$ is positive semi-definite. Because $\mathbb{C}^{N}=\mathcal{C}\left(\left[\mathbf{H}_{1 i}^{H}, \cdots, \mathbf{H}_{K i}^{H}\right]\right) \oplus$ $\mathcal{C}\left(\left\{\mathbf{u}_{l}\right\}_{l=1}^{N-m}\right), \mathbf{Q}_{i}^{\prime}$ is positive semi-definite. 
Then, the rate of user $j(\neq i)$ achieved by $\left\{\mathbf{Q}_{1}, \cdots, \mathbf{Q}_{i}^{\prime \prime}, \cdots, \mathbf{Q}_{K}\right\}$ is given by

$$
\begin{aligned}
& R_{j}\left(\mathbf{Q}_{1}, \cdots, \mathbf{Q}_{i}^{\prime \prime}, \cdots, \mathbf{Q}_{K}\right) \\
& =\log _{2}\left|\mathbf{I}+\left(\mathbf{I}+\sum_{k \neq i, k \neq j} \mathbf{H}_{j k} \mathbf{Q}_{k} \mathbf{H}_{j k}^{H}+\mathbf{H}_{j i} \mathbf{Q}_{i}^{\prime \prime} \mathbf{H}_{j i}^{H}\right)^{-1} \mathbf{H}_{j j} \mathbf{Q}_{j} \mathbf{H}_{j j}^{H}\right| \\
& =\log _{2}\left|\mathbf{I}+\left(\boldsymbol{\Psi}_{j}+\delta \mathbf{H}_{j i} \mathbf{v} \mathbf{v}^{H} \mathbf{H}_{j i}^{H}\right)^{-1} \mathbf{H}_{j j} \mathbf{Q}_{j} \mathbf{H}_{j j}^{H}\right| \\
& \stackrel{(c)}{=} \log _{2}\left|\mathbf{I}+\mathbf{\Psi}_{j}^{-1} \mathbf{H}_{j j} \mathbf{Q}_{j} \mathbf{H}_{j j}^{H}\right| \\
& =R_{j}\left(\left\{\mathbf{Q}_{1}, \cdots, \mathbf{Q}_{i}^{\prime}, \cdots, \mathbf{Q}_{K}\right\}\right),
\end{aligned}
$$

where $(c)$ holds from the construction of $\mathbf{v}$. On the other hand, the rate of user $i$ achieved by $\left\{\mathbf{Q}_{1}, \cdots, \mathbf{Q}_{i}^{\prime \prime}, \cdots, \mathbf{Q}_{K}\right\}$ is given by

$$
\begin{aligned}
& R_{i}\left(\left\{\mathbf{Q}_{1}, \cdots, \mathbf{Q}_{i}^{\prime \prime}, \cdots, \mathbf{Q}_{K}\right\}\right) \\
& =\log _{2}\left|\mathbf{I}+\boldsymbol{\Phi}_{i}^{-1} \mathbf{H}_{i i} \mathbf{Q}_{i}^{\prime \prime} \mathbf{H}_{i i}^{H}\right| \\
& \stackrel{(d)}{=} \log _{2}\left|\boldsymbol{\Phi}_{i}+\mathbf{H}_{i i} \mathbf{Q}_{i}^{\prime \prime} \mathbf{H}_{i i}^{H}\right|-\log _{2}\left|\boldsymbol{\Phi}_{i}\right| \\
& =\log _{2}\left|\boldsymbol{\Phi}_{i}+\mathbf{H}_{i i}\left(\mathbf{Q}_{i}^{\prime}+\delta \mathbf{v} \mathbf{v}^{H}\right) \mathbf{H}_{i i}^{H}\right|-\log _{2}\left|\boldsymbol{\Phi}_{i}\right| \\
& \stackrel{(e)}{>} \log _{2}\left|\boldsymbol{\Phi}_{i}+\mathbf{H}_{i i} \mathbf{Q}_{i}^{\prime} \mathbf{H}_{i i}^{H}\right|-\log _{2}\left|\boldsymbol{\Phi}_{i}\right| \\
& =\log _{2}\left|\mathbf{I}+\mathbf{\Phi}_{i}^{-1} \mathbf{H}_{i i} \mathbf{Q}_{i}^{\prime} \mathbf{H}_{i i}^{H}\right| \\
& =R_{i}\left(\left\{\mathbf{Q}_{1}, \cdots, \mathbf{Q}_{i}^{\prime}, \cdots, \mathbf{Q}_{K}\right\}\right),
\end{aligned}
$$

where $(d)$ follows from the fact of $\left|\mathbf{I}+\mathbf{A}^{-1} \mathbf{B}\right|=\left|\mathbf{A}^{-1}\right||\mathbf{A}+\mathbf{B}|$ and $(e)$ holds by Lemma 1. Based on (11), (12), (16), and (17), we have

$$
\begin{aligned}
& R_{i}\left(\left\{\mathbf{Q}_{1}, \cdots, \mathbf{Q}_{i}^{\prime \prime}, \cdots, \mathbf{Q}_{K}\right\}\right)>R_{i}\left(\left\{\mathbf{Q}_{1}, \cdots, \mathbf{Q}_{i}, \cdots, \mathbf{Q}_{K}\right\}\right), \\
& R_{j}\left(\left\{\mathbf{Q}_{1}, \cdots, \mathbf{Q}_{i}^{\prime \prime}, \cdots, \mathbf{Q}_{K}\right\}\right)=R_{j}\left(\left\{\mathbf{Q}_{1}, \cdots, \mathbf{Q}_{i}, \cdots, \mathbf{Q}_{K}\right\}\right)
\end{aligned}
$$

for all $j \neq i$, and this contradicts our assumption of Paretooptimality of $\left\{\mathbf{Q}_{1}, \cdots, \mathbf{Q}_{i}, \cdots, \mathbf{Q}_{K}\right\}$. Therefore, $\mathcal{C}\left(\mathbf{Q}_{i}\right) \subseteq$ $\mathcal{C}\left(\left[\mathbf{H}_{1 i}^{H}, \cdots, \mathbf{H}_{K i}^{H}\right]\right)$ for the $K$-pair Gaussian $\left(N, M_{1}, \cdots, M_{K}\right)$ MIMO interference channel.

Next, suppose that $\mathbf{Q}_{i}$ satisfies (5) but $\operatorname{tr}\left(\mathbf{Q}_{i}\right)<P_{i}$. When $N \geq$ $\sum_{i} M_{i}$, there almost surely exists a vector $\mathbf{v}$ such that $\mathbf{H}_{i i} \mathbf{v} \neq \mathbf{0}$ and $\mathbf{H}_{j i} \mathbf{v}=\mathbf{0}$ for all $j \neq i$ by the same argument as before. Now, let

$$
\overline{\mathbf{Q}}_{i}=\mathbf{Q}_{i}+\bar{\delta} \mathbf{v} \mathbf{v}^{H},
$$

where $\bar{\delta}$ is set to be $\bar{\delta}=\frac{1}{\operatorname{tr}\left(\mathbf{v} \mathbf{v}^{H}\right)}\left(P_{i}-\operatorname{tr}\left(\mathbf{Q}_{i}\right)\right)$ so that $\operatorname{tr}\left(\overline{\mathbf{Q}}_{i}\right)=$ $P_{i}$. Then, the rate of receiver $j(\neq i)$ does not change as in (16) whereas the rate of receiver $i$ increases by the same argument as in (17). Hence, when $N \geq \sum_{i} M_{i}$, each transmitter should use its maximum transmit power for Pareto-optimality.

Finally, when $N<\sum_{i=1}^{K} M_{i}, \mathcal{C}\left(\left[\mathbf{H}_{1 i}^{H}, \cdots, \mathbf{H}_{K i}^{H}\right]\right)=\mathbb{C}^{N}$ for randomly realized channel matrices and (5) holds trivially.

Lemma 1 Under the same conditions as in Theorem 1, we have

$\log \left|\boldsymbol{\Phi}_{i}+\mathbf{H}_{i i}\left(\mathbf{Q}_{i}^{\prime}+\delta \mathbf{v} \mathbf{v}^{H}\right) \mathbf{H}_{i i}^{H}\right|>\log \left|\mathbf{\Phi}_{i}+\mathbf{H}_{i i} \mathbf{Q}_{i}^{\prime} \mathbf{H}_{i i}^{H}\right|$.

Proof: First, by taking the difference of the two matrices

$$
\begin{aligned}
& \left(\boldsymbol{\Phi}_{i}+\mathbf{H}_{i i}\left(\mathbf{Q}_{i}^{\prime}+\delta \mathbf{v} \mathbf{v}^{H}\right) \mathbf{H}_{i i}^{H}\right)-\left(\boldsymbol{\Phi}_{i}+\mathbf{H}_{i i} \mathbf{Q}_{i}^{\prime} \mathbf{H}_{i i}^{H}\right) \\
& =\delta \mathbf{H}_{i i} \mathbf{v} \mathbf{v}^{H} \mathbf{H}_{i i}^{H} \succcurlyeq \mathbf{0},
\end{aligned}
$$

we can see that $\boldsymbol{\Phi}_{i}+\mathbf{H}_{i i}\left(\mathbf{Q}_{i}^{\prime}+\delta \mathbf{v} \mathbf{v}^{H}\right) \mathbf{H}_{i i}^{H} \succcurlyeq \boldsymbol{\Phi}_{i}+\mathbf{H}_{i i} \mathbf{Q}_{i}^{\prime} \mathbf{H}_{i i}^{H}$. This implies [13, p.471],

$$
\lambda_{k}^{\prime \prime} \geq \lambda_{k}^{\prime}>0, \quad \forall k .
$$

where $\lambda_{k}^{\prime \prime}$ and $\lambda_{k}^{\prime}$ are the $k$-th largest eigenvalues of $\boldsymbol{\Phi}_{i}+\mathbf{H}_{i i}\left(\mathbf{Q}_{i}^{\prime}+\right.$ $\left.\delta \mathbf{v v}{ }^{H}\right) \mathbf{H}_{i i}^{H}$ and $\boldsymbol{\Phi}_{i}+\mathbf{H}_{i i} \mathbf{Q}_{i}^{\prime} \mathbf{H}_{i i}^{H}$, respectively. The strict positivity of the eigenvalues is from the strict positive definiteness of the two matrices because of the added identity matrix in $\boldsymbol{\Phi}_{i}$. Next, consider the difference of the traces of the two matrices:

$$
\begin{aligned}
& \operatorname{tr}\left(\boldsymbol{\Phi}_{i}+\mathbf{H}_{i i}\left(\mathbf{Q}_{i}^{\prime}+\delta \mathbf{v} \mathbf{v}^{H}\right) \mathbf{H}_{i i}^{H}\right)-\operatorname{tr}\left(\boldsymbol{\Phi}_{i}+\mathbf{H}_{i i} \mathbf{Q}_{i}^{\prime} \mathbf{H}_{i i}^{H}\right) \\
& =\delta \operatorname{tr}\left(\mathbf{H}_{i i} \mathbf{v} \mathbf{v}^{H} \mathbf{H}_{i i}^{H}\right)=\delta\left\|\mathbf{H}_{i i} \mathbf{v}\right\|^{2}>0
\end{aligned}
$$

where (22) holds since $\mathbf{H}_{i i} \mathbf{v} \neq \mathbf{0}$ by the construction of $\mathbf{v}$. From (21) and (22), at least one eigenvalue $\lambda_{k}^{\prime \prime}$ is strictly larger than $\lambda_{k}^{\prime}$ since the trace of a matrix is the sum of its eigenvalues. Therefore, we have

$$
\begin{aligned}
& \prod_{k=1}^{M_{i}} \lambda_{k}^{\prime \prime}>\prod_{k=1}^{M_{i}} \lambda_{k}^{\prime} \\
\Leftrightarrow & \left|\boldsymbol{\Phi}_{i}+\mathbf{H}_{i i}\left(\mathbf{Q}_{i}^{\prime}+\delta \mathbf{v} \mathbf{v}^{H}\right) \mathbf{H}_{i i}^{H}\right|>\left|\boldsymbol{\Phi}_{i}+\mathbf{H}_{i i} \mathbf{Q}_{i}^{\prime} \mathbf{H}_{i i}^{H}\right| .
\end{aligned}
$$

Finally, (20) holds by the monotonicity of logarithm.

Note that in the case of $M_{i}=1$ for all $i$, Theorem 1 reduces to the statement that any Pareto-optimal beam vector is a linear combination of the (complex conjugated) channel vectors from the transmitter to all receivers. Thus, the result in Theorem 1 is a MIMO extension of the result for Pareto-optimal coordinated beamforming in MISO interference channels by Jorswieck et al. [2].

\section{PARAMETERIZATION FOR PARETO-OPTIMAL TRANSMIT SIGNAL COVARIANCE MATRICES}

In this section, we provide a concrete parameterization for Paretooptimal transmit signal covariance matrices based on Theorem 1. We mainly consider the case when $N \geq \sum_{i=1}^{K} M_{i}$, even though the result in this section can be applied to $N<\sum_{i=1}^{K} M_{i}$.

Due to (5), any Pareto-optimal transmit signal covariance matrix $\mathbf{Q}_{i}^{\star}$ can be expressed as

$$
\mathbf{Q}_{i}^{\star}=\left[\mathbf{H}_{1 i}^{H}, \cdots, \mathbf{H}_{K i}^{H}\right] \mathbf{X}_{i}\left[\mathbf{H}_{1 i}^{H}, \cdots, \mathbf{H}_{K i}^{H}\right]^{H},
$$

where $\mathbf{X}_{i} \in \mathbb{S}_{+}^{\left(\sum_{i} M_{i}\right) \times\left(\sum_{i} M_{i}\right)}$ with rank less than or equal to $M_{i}$. Note that the matrix $\left[\mathbf{H}_{1 i}^{H}, \cdots, \mathbf{H}_{K i}^{H}\right]$ has size $N \times\left(\sum_{i} M_{i}\right)$ and it has full column rank almost surely for randomly realized channel matrices. Let the skinny QR factorization of $\left[\mathbf{H}_{1 i}^{H}, \cdots, \mathbf{H}_{K i}^{H}\right]$ be

$$
\left[\mathbf{H}_{1 i}^{H}, \cdots, \mathbf{H}_{K i}^{H}\right]=\mathbf{\Upsilon}_{i} \mathbf{R}_{i},
$$

where $\boldsymbol{\Upsilon}_{i}$ is a $N \times \sum_{i} M_{i}$ matrix with orthonormal columns and $\mathbf{R}_{i}$ is an upper triangular matrix with size $\left(\sum_{i} M_{i}\right) \times\left(\sum_{i} M_{i}\right)$. With (25), the condition (5) can be rewritten as

$$
\mathbf{Q}_{i}^{\star}=\Upsilon_{i} \mathbf{X}_{i}^{\prime} \mathbf{\Upsilon}_{i}^{H}
$$

where $\mathbf{X}_{i}^{\prime} \in \mathbb{S}_{+}^{\left(\sum_{i} M_{i}\right) \times\left(\sum_{i} M_{i}\right)}$ with rank less than or equal to $M_{i}$. Since $\mathbf{X}_{i}^{\prime}$ is Hermitian, its spectral decomposition is given by

$$
\mathbf{X}_{i}^{\prime}=\mathbf{U}_{i} \boldsymbol{\Lambda}_{i} \mathbf{U}_{i}^{H}
$$


where $\mathbf{U}_{i}$ is a $\left(\sum_{i} M_{i}\right) \times M_{i}$ matrix with orthonormal columns and $\boldsymbol{\Lambda}_{i}=\operatorname{diag}\left(\lambda_{i 1}, \cdots, \lambda_{i M_{i}}\right)$ is a $M_{i} \times M_{i}$ diagonal matrix with nonnegative diagonal elements. Therefore, any Pareto-optimal transmit signal covariance matrix $\mathbf{Q}_{i}^{\star}$ can be expressed by

$$
\mathbf{Q}_{i}^{\star}=\Upsilon_{i} \mathbf{U}_{i} \boldsymbol{\Lambda}_{i} \mathbf{U}_{i}^{H} \mathbf{\Upsilon}_{i}^{H},
$$

which turns out to be a spectral decomposition of $\mathbf{Q}_{i}^{\star}$ since $\left(\mathbf{\Upsilon}_{i} \mathbf{U}_{i}\right)^{H}$ $\left(\boldsymbol{\Upsilon}_{i} \mathbf{U}_{i}\right)=\mathbf{I}$ and $\boldsymbol{\Lambda}_{i}$ is diagonal. Note that $\boldsymbol{\Upsilon}_{i}$ is given and fixed when the channel matrices are given. Furthermore, in the case of $N \geq \sum_{i} M_{i}$, we have the full power condition (6). The full power condition is expressed by

$$
\begin{aligned}
P_{i} & =\operatorname{tr}\left(\mathbf{Q}_{i}^{\star}\right)=\operatorname{tr}\left(\mathbf{\Upsilon}_{i} \mathbf{U}_{i} \boldsymbol{\Lambda}_{i} \mathbf{U}_{i}^{H} \mathbf{\Upsilon}_{i}^{H}\right) \\
& =\operatorname{tr}\left(\boldsymbol{\Lambda}_{i} \mathbf{U}_{i}^{H} \mathbf{\Upsilon}_{i}^{H} \mathbf{\Upsilon}_{i} \mathbf{U}_{i}\right), \quad\left(\mathbf{\Upsilon}_{i} \mathbf{U}_{i}\right)^{H}\left(\mathbf{\Upsilon}_{i} \mathbf{U}_{i}\right)=\mathbf{I} \\
& =\operatorname{tr}\left(\boldsymbol{\Lambda}_{i}\right)=\sum_{k=1}^{M_{i}} \lambda_{i k},
\end{aligned}
$$

where $\lambda_{i k} \geq 0$ for all $k$. Thus, any Pareto-optimal transmit signal covariance matrix can be parameterized by $\mathbf{U}_{i}$ and $\boldsymbol{\Lambda}_{i}$ with respective constraints of $\mathbf{U}_{i}^{H} \mathbf{U}_{i}=\mathbf{I}$ and $\operatorname{tr}\left(\boldsymbol{\Lambda}_{i}\right)=P_{i}$. Here, $\mathbf{U}_{i}$ 's with constraint $\mathbf{U}_{i}^{H} \mathbf{U}_{i}=\mathbf{I}$ form a special subset in $\mathbb{C}^{\left(\sum_{i} M_{i}\right) \times M_{i}}$ called the Stiefel manifold $V_{\sum_{i} M_{i}, M_{i}}$ [14]. We summarize the result in the following theorem.

Theorem 2 Any Pareto-optimal transmit signal covariance matrix at transmitter $i$ for the $K$-pair Gaussian $\left(N, M_{1}, \cdots, M_{K}\right)$ MIMO interference channel with $N \geq \sum_{i} M_{i}$ is parameterized by the product manifold $\mathcal{M}_{i}$ :

$$
\mathcal{M}_{i}:=V_{\sum_{i} M_{i}, M_{i}} \times \mathcal{H}_{M_{i}},
$$

where $V_{\sum_{i} M_{i}, M_{i}}$ is the Stiefel manifold of orthonormal $M_{i}$-frames in $\mathbb{C}^{\sum_{i} M_{i}}$ and $\mathcal{H}_{M_{i}}$ is a subset in the first quadrant of a hyperplane in the Euclidean space $\mathbb{R}^{M_{i}}$ defined by

$$
\mathcal{H}_{M_{i}}:=\left\{\left(\lambda_{1}, \cdots, \lambda_{M_{i}}\right): \lambda_{i} \geq 0 \text { and } \sum_{i} \lambda_{i}=P_{i}\right\} .
$$

Proof: Combining Theorem 1 and equations (26), (27), (28) and (29), we have the result.

Note that the new beam search space or parameter space $\mathcal{M}_{i}$ is an embedded submanifold of the original beam search space $\mathcal{Q}_{i}$. The main advantage of the parameterization in Theorem 2 is that the dimension of the parameter space does not depend on the number of transmit antennas but only on the number of receive antennas, while Pareto-optimality is not lost. The proposed parameterization significantly reduces the search space dimension as compared to the original search space $\mathcal{Q}_{i}$ when $N \gg \sum_{i} M_{i}$, and thus the proposed parameterization is useful for emerging cellular downlink systems with massive MIMO technologies [15,16]. Furthermore, the parameterization in Theorem 2 enables us to construct efficient beam design algorithms by exploiting the rich geometrical structure of Stiefel manifolds and hyperplanes. (For the advantages of the proposed parameterization, please see [1].)

\section{NUMERICAL RESULTS}

In this section, we provide some numerical results based on the results in the previous section. We considered the weighted sum rate maximization problem in the $K$-pair Gaussian $\left(N, M_{1}, \cdots, M_{K}\right)$ MIMO interference channel, given by

$$
\max _{\left\{\left(\mathbf{U}_{i}, \mathbf{\Lambda}_{i}\right)\right\}} \sum_{i=1}^{K} w_{i} R_{i}
$$

where the optimization variables are the matrices $\left\{\mathbf{U}_{i}\right\}$ with orthonormal columns and the diagonal power loading matrices $\left\{\boldsymbol{\Lambda}_{i}\right\}$ in Theorem 2. We developed an efficient algorithm to solve the weighted sum rate maximization problem by exploiting the geometrical properties of Stiefel manifolds [1] and applied the algorithm to the 2-pair case. The result is shown in Fig. 1. Here, we considered the 2-pair Gaussian $(5,2,2)$ MIMO interference channel with $\mathrm{SNR}=3 \mathrm{~dB}$. In this example, the beam design space with our new parameterization is the product of a stiefel manifold $V_{4,2}$ $(4 \times 2$ unitary matrices) and an 1-dimensional hyperplane (line), whereas the convential beam search space is the set of $5 \times 5$ positivesemidefinite matrices. The obtained rate-tuples by the proposed algorithm with different weighting factors and the rate-tuples by eigenbeamforming (SVD based beamforming) and zero-forcing beamforming are shown in the figure. Although we cannot guarantee that the obtained rate-tuples are on the Pareto boundary of the achievable rate region (the derived condition is a necessary condition), the rate-tuples obtained by a beam design algorithm based on the proposed parameterization $\mathcal{M}_{1} \times \mathcal{M}_{2}$ are much more likely to be on the Pareto boundary than those obtained by a beam design algorithm based on the original high dimensional search space $\mathcal{Q}_{1} \times \mathcal{Q}_{2}$. This is because local optima and peculiar behaviors are more likely to happen in higher dimensional spaces. (For detail on the beam design algorithm with the proposed parameterization, please see [1].)

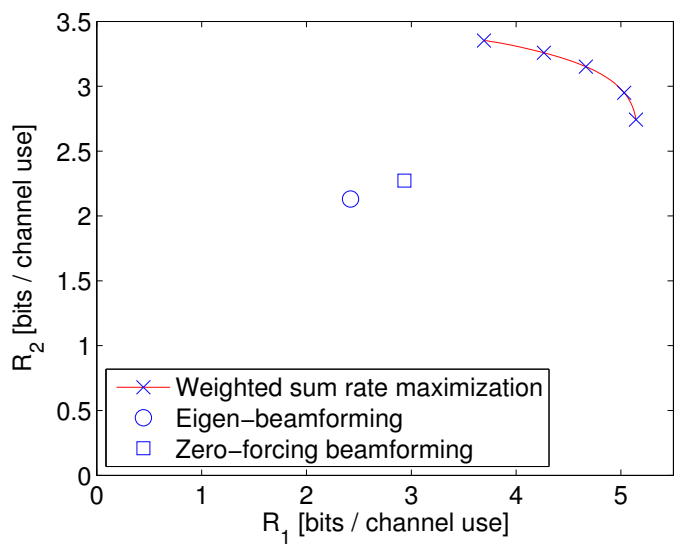

Fig. 1. Weighted sum rate maximization for the 2-pair Gaussian $(5,2,2)$ MIMO interference channel.

\section{CONCLUSION}

In this paper, we have provided a necessary condition for Paretooptimal transmit signal covariance matrices in the $K$-pair Gaussian $\left(N, M_{1}, \cdots, M_{K}\right)$ MIMO interference channel and a parameterization for the beam search space not losing Pareto-optimality. The proposed parameter space is given by an embedded submanifold of the original beam search space and described by the product manifold of a Stiefel manifold and a subset of a hyperplane. The proposed parameterization is especially useful when the number of transmit antennas is much larger than that of receive antennas, as in upcoming cellular networks adopting massive MIMO technologies. 


\section{REFERENCES}

[1] J. Park and Y. Sung, "On the Pareto-optimal beam structure and design for multi-user MIMO interference channels," ArXiv pre-print cs.IT/1211.4213, Nov. 2012.

[2] E. Jorswieck, E. Larsson, and D. Danev, "Complete characterization of the pareto boundary for the MISO interference channel," IEEE Trans. Signal Process., vol. 56, no. 10, pp. $5292-$ 5296, Oct. 2008.

[3] E. Björnson, R. Zakhour, D. Gesbert, and B. Ottersten, "Cooperate multicell precoding: Rate region characterization and distributed strategies with instantaneous and statistical CSI," IEEE Trans. Signal Process., vol. 58, no. 8, pp. 4298 - 4310, Aug. 2010.

[4] R. Zhang and S. Cui, "Cooperative interference management with MISO beamforming," IEEE Trans. Signal Process., vol. 58 , no. 10 , pp. $5450-5458$, Oct. 2010.

[5] R. Zakhour and D. Gesbert, "Distributed multicell-MISO precoding using the layered virtual SINR framework," IEEE Trans. Wireless Commun., vol. 9, no. 8, pp. 2444 - 2448, Aug. 2010.

[6] R. Mochaourab and E. Jorswieck, "Optimal beamforming in interference networks with perfect local channel information," IEEE Trans. Signal Process., vol. 59, no. 3, pp. 1128 - 1141, Mar. 2011.

[7] X. Shang, B. Chen, and H. V. Poor, "Multiuser MISO interference channels with single-user detection: Optimality of beamforming and the achievable rate region," IEEE Trans. Inf. Theory, vol. 57, no. 7, pp. 4255 - 4273, Jul. 2011.

[8] J. Park, G. Lee, Y. Sung, and M. Yukawa, "Coordinated beamforming with relaxed zero forcing: The sequential orthogonal projection combining method and rate control," ArXiv preprint cs.IT/1203.1758, Mar. 2012.

[9] E. Björnson, M. Bengtsson, and B. Ottersten, "Pareto characterization of the multicell MIMO performance region with simple receivers," IEEE Trans. Signal Process., vol. 60, no. 8, pp. 4464 - 4469, Aug. 2012.

[10] P. Cao, E. Jorswieck, and S. Shi, "On the Pareto boundary for the two-user single-beam MIMO interference channel," ArXiv pre-print cs.IT/1202.5474, Feb. 2012.

[11] Z. Chen, S. A. Vorobyov, C.-X. Wang, and J. Thompson, "Pareto region characterization for rate control in MIMO interference systems and Nash bargaining," IEEE Trans. Autom. Control, vol. 57, no. 12, pp. 3203 - 3208, Dec. 2012.

[12] J. Lindblom, E. Larsson, and E. Jorswieck, "Parametrization of the MISO IFC rate region: The case of partial channel state information," IEEE Trans. Wireless Commun., vol. 9, no. 2, pp. $500-504$, Feb. 2010.

[13] R. A. Horn and C. R. Johnson, Matrix Analysis, Cambridge University Press, Cambridge, UK, 1985.

[14] R. A. Absil, R. Mahony, and R. Sepulchre, Optimization Algorithms on Matrix Manifolds, Princeton University Press, Princeton, NJ, 2007.

[15] T. L. Marzetta, "Noncooperative celular wireless with unlimited number of base station antennas," IEEE Trans. Wireless Commun., vol. 9, no. 11, pp. 3590 - 3600, Nov. 2010.
[16] F. Rusek, D. Persson, B. K. Lau, E. G. Larsson, T. L. Marzetta, O. Edfor, and F. Tufvesson, "Scaling up MIMO: Opportunities and challenges with very large arrays," ArXiv pre-print cs.IT/1201.3210, Jan. 2012. 